\title{
ACM Multimedia BioMedia 2020 Grand Challenge Overview
}

\author{
Steven A. Hicks* \\ SimulaMet, Norway \\ Trine B. Haugen \\ Oslo Metropolitan University, Norway \\ Pål Halvorsen*
SimulaMet, Norway
}

\author{
Vajira Thambawita* \\ SimulaMet, Norway
}

Jorunn M. Andersen

Oslo Metropolitan University, Norway

\author{
Hugo L. Hammer ${ }^{\dagger}$ \\ Oslo Metropolitan University, Norway \\ Oliwia Witczak \\ Oslo Metropolitan University, Norway
}

\begin{abstract}
The BioMedia 2020 ACM Multimedia Grand Challenge is the second in a series of competitions focusing on the use of multimedia for different medical use-cases. In this year's challenge, participants are asked to develop algorithms that automatically predict the quality of a given human semen sample using a combination of visual, patient-related, and laboratory-analysis-related data. Compared to last year's challenge, participants are provided with a fully multimodal dataset (videos, analysis data, study participant data) from the field of assisted human reproduction. The tasks encourage the use of the different modalities contained within the dataset and finding smart ways of how they may be combined to further improve prediction accuracy. For example, using only video data or combining video data and patient-related data. The ground truth was developed through a preliminary analysis done by medical experts following the World Health Organization's standard for semen quality assessment. The task lays the basis for automatic, real-time support systems for artificial reproduction. We hope that this challenge motivates multimedia researchers to explore more medical-related applications and use their vast knowledge to make a real impact on people's lives.
\end{abstract}

\section{CCS CONCEPTS}

- Applied computing $\rightarrow$ Consumer health; Health informatics; • Computing methodologies $\rightarrow$ Supervised learning; • Information systems $\rightarrow$ Summarization.

\section{KEYWORDS}

Male fertility, Semen Analysis, Spermatozoa, Machine Learning, Artificial Intelligence

\section{ACM Reference Format:}

Steven A. Hicks, Vajira Thambawita, Hugo L. Hammer, Trine B. Haugen, Jorunn M. Andersen, Oliwia Witczak, Pål Halvorsen, and Michael A. Riegler.

\footnotetext{
*Also affiliated with Oslo Metropolitan University, Norway

$\dagger$ Also affiliated with SimulaMet, Norway

Permission to make digital or hard copies of part or all of this work for personal or classroom use is granted without fee provided that copies are not made or distributed for profit or commercial advantage and that copies bear this notice and the full citation on the first page. Copyrights for third-party components of this work must be honored.

For all other uses, contact the owner/author(s).

MM '20, October 12-16, 2020, Seattle, WA, USA

(C) 2020 Copyright held by the owner/author(s)

ACM ISBN 978-1-4503-7988-5/20/10.

https://doi.org/10.1145/3394171.3416287
}

\author{
Michael A. Riegler \\ SimulaMet, Norway
}

2020. ACM Multimedia BioMedia 2020 Grand Challenge Overview. In Proceedings of the 28th ACM International Conference on Multimedia (MM '20), October 12-16, 2020, Seattle, WA, USA. ACM, New York, NY, USA, 4 pages. https://doi.org/10.1145/3394171.3416287

\section{INTRODUCTION}

The BioMedia 2020 ACM Multimedia Grand Challenge ${ }^{1}$ is a competition that aims to introduce multimedia researchers to different medical-related tasks that solve real-world challenges. Last year [10], the goal was to automatically analyze images and videos taken from routine investigations of the human digestive tract in order to identify disease, anatomical landmarks, or other relevant findings. This year, we move the focus to assisted reproduction and how multimedia researchers can aid in the development of tools that help determine the quality of a given semen sample. This challenge is the next version of the 2019 Medico: Multimedia for Medicine task, which has previously been held at MediaEval Benchmark [10]. Furthermore, the challenge was discussed in the tutorial Medical Multimedia Systems and Applications [8] at ACM Multimedia 2019, where attendees showed much interest in this multimodal problem. As the task requires an analysis of a combination of different data modalities, we find that it is a perfect fit for ACM Multimedia and that there is a high chance of making a real impact on the field of human reproduction.

Assessment of semen is usually performed during the early stages of male infertility testing. This is mostly a manual process, where trained clinicians inspect semen samples through a microscope and count the number of different sperms with certain qualities, like the number of moving or not moving sperm, to evaluate quality [4]. As one might expect, this is a tedious and time-consuming process that could greatly benefit from some automation. Furthermore, due to the subjective nature of manually inspecting large numbers of moving sperm, there is a large interand intra-observer variability between and within clinics. Having an algorithm that can give consistent results for any given semen sample would be of great benefit. This year, we present four different tasks, each targeting a different aspect of sperm quality assessment. The first two tasks relate to predicting common measurements used for general fertility testing, specifically the motility (movement) and morphology (shape and structure) of the spermatozoa (living sperm). These two tasks encourage participants to combine all available data modalities and data sources to make predictions. Besides

\footnotetext{
${ }^{1}$ https://www.biomediachallenge.com
} 

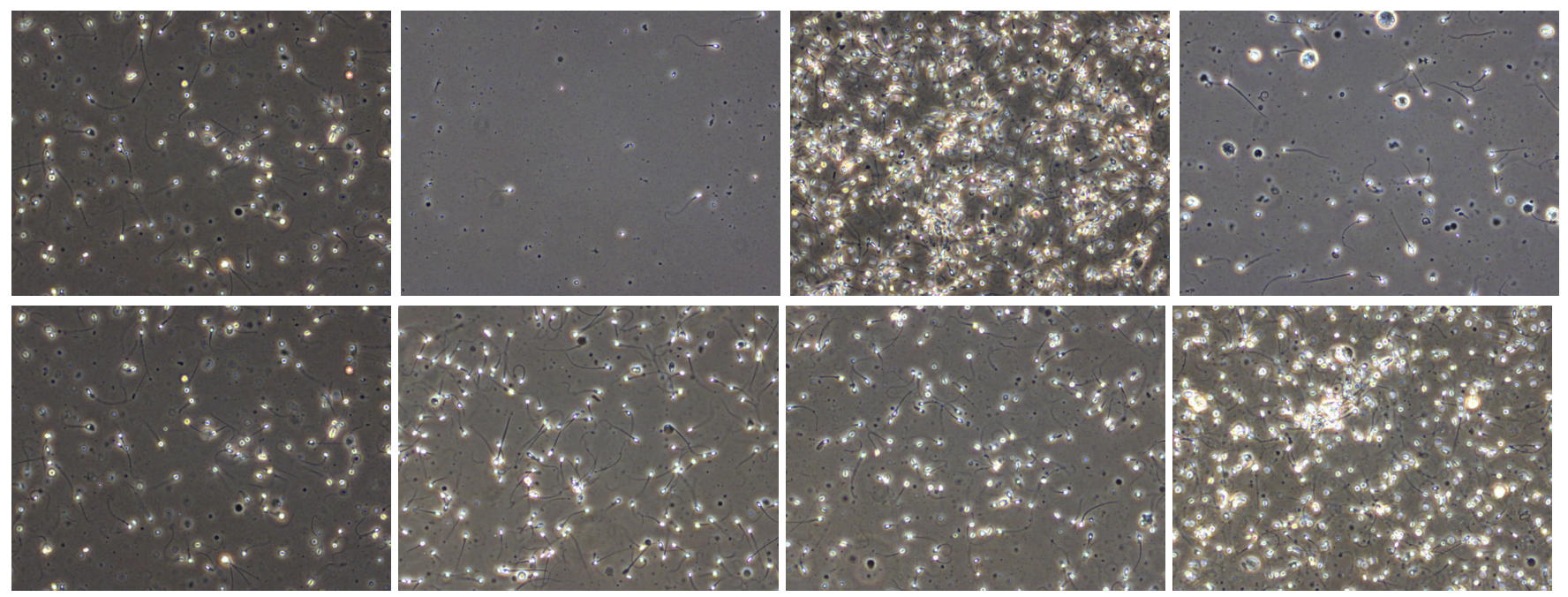

Figure 1: Sample video frames taken from the dataset. Note the variety of number of cells in the different video samples.

the quantitative and measurable tasks, we also have a more subjective part (optional). Participants are asked to come up with a tool that can help medical doctors choose an ideal sperm for assisted reproduction. This could be as simple as providing an interface to aid in manual tracking to more advanced methods such as unsupervised spermatozoa tracking. The winner of this sub challenge will be chosen by the majority vote of three andrology experts through testing the tool in a clinical setting. The assessment will primarily be based on the usefulness and novelty of the idea.

We provide a dataset consisting of 85 videos, a set of sperm characteristics (such as hormones or fatty acids data), anonymized study participants-related data, and preliminary analysis data collected according to the World Health Organization's standards [20] (ground truth for sperm quality assessment). It is a typical assumption that visual analysis, as provided by the computer vision and medical image processing communities today, is capable of already providing viable and practical approaches to healthcare multimedia challenges. Automatic analysis of human semen is an active field of research supported by several studies on the topic $[1,3,6,7,11,15,17]$. However, a common theme is that approaches usually focus on one modality and do not incorporate other data sources into their analysis. Although we concede that these methods are indeed essential contributors to promising approaches, we realize the limitations of analyzing images and videos independently in medical fields, such as endoscopy or ultrasound, because of the complexity and needs of medical experts and patients. Neither does it make enough use of the multitude of additional information sources, including sensors and temporal information. The challenge can be seen as very challenging and hard to solve. Due to its novel use-case, we hope to motivate many researchers to look into the field of medical multimedia [16]. Performing research that can have a societal impact will be an essential part of multimedia research. We hope that the challenge can help raise awareness of the topic and provide exciting and meaningful use-cases to researchers.

\section{DATASET DETAILS}

The challenge uses an open dataset called VISEM [9], which contains data from 85 free-willing male participants aged 18 years or older. For each participant, the dataset presents a set of data collected from a standard semen analysis, which includes a video of the spermatozoa under a microscope, a sperm fatty acid profile, the fatty acid composition of serum phospholipids, participants-related data, and set of preliminary analysis data collected in accordance to the WHO guidelines. The dataset contains a wide variety of samples with varying quality. As we can see in Figure 1, samples vary from containing just a few cells to containing a lot.

The dataset contains over 35 gigabytes of videos, with each video lasting between two to seven minutes. Each video has a resolution of $640 \times 480$ and runs at 50 frames-per-second. The dataset contains six CSV-files, a description file, and a folder containing the videos. Each video file's name contains the videos ID, the date it was recorded, and a small description. Then, the end of the filename contains the code of the person who assessed the video. Furthermore, VISEM contains five CSV-files for each category of sensor/patient-related data, a CSV-file with the IDs linked to each their video, and a text file containing descriptions of some of the columns of the CSV-files. One row in each CSV-file represents one participant. In addition to the videos, we provide pre-extracted features for all videos in the dataset.

The features contain a collection of visual features (Tamura, JCD, Edge histogram, and color histogram $[14,18])$. All visual features were extracted from the first two frames of every second for sixty seconds of each video. To extract the features, we use the opensource library LIRE [14]. For the final evaluation, participants were asked to use three-fold cross-validation on a pre-defined split of the dataset to calculate the final results. The VISEM dataset [9] is publicly available $\mathrm{e}^{2}$ for participants and other multimedia researchers without any restriction. All study participants agreed to donate their data for science and provided the necessary consent for us to 
be able to distribute the data (checked and approved by Norwegian data authority and ethical committee).

\section{TASKS AND EVALUATION METRICS}

This year, we present four different tasks, of which two are required to participate in the challenge. For evaluating the first two tasks, we primarily use the mean absolute error (MAE) to compare submissions and report a series of other metrics that the participants may decide to use in their report. For both tasks, we asked participants to perform video analysis over single frame analysis. This is important since single frame-based analysis will not be able to catch the movement of the spermatozoa, which contains essential information to perform the predictions. For the optional challenges, we use manual evaluation with the help of three experts within human reproduction. The experts will deliver a subjective assessment of the submissions based on the delivered result's clinical viability. The script used to evaluate all quantitative submissions is published online for transparency and reproducibility ${ }^{3}$.

\subsection{Prediction of Motility Task}

One of the most common measurements used to evaluate a person's semen quality is looking at the motility of the spermatozoa contained within. Healthy spermatozoon should move at a rapid pace in a forward trajectory. If not, it may be a sign that the sperm will not perform as well. This task aims to predict the percentage of progressive, non-progressive, and immotile sperm in a given semen sample. Motility is the ability of an organism to move independently. Where a progressive spermatozoon can "move forward", a non-progressive would move in circles without progression. An immotile sperm does not move at all. The number of progressive, non-progressive, and immotile spermatozoon should sum up to $100 \%$, meaning every sperm in the sample should be counted.

Submissions to this task should be a CSV file containing the percentage of progressive, non-progressive, and immotile spermatozoon for a given sample. The prediction needs to be performed sample wise resulting in one set of predictions per video sample. No sperm tracking or bounding boxes are required to solve the task. The goal is to maximize the algorithm's performance in terms of prediction accuracy. In this context, accuracy will be evaluated based on the achieved MAE, which is calculated over three-fold cross-validation on the provided test dataset split.

\subsection{Prediction of Morphology Task}

Morphology is a branch of biology dealing with the study of an organism's form and structural features. In the context of semen, doctors often examine the three parts that make up a spermatozoon, which includes the head, midpiece, and tail, to see if there are any defects. Defects are common and could hamper the spermatozoa ability to move, making it less likely to fertilize. This task should predict the percentage of sperm with head defects, midpiece defects, and tail defects.

Similar to the motility task, the submission to this task should be a CSV file containing the percentage of cells with head defects, percentage of cells with midpiece defects, and the percentage of cells with tail defects. Morphology analysis only requires sample

\footnotetext{
${ }^{3}$ https://github.com/simula/biomedia-2020-submission-evaluation
}

wise prediction resulting in one value per sample per predicted attribute, meaning one set of predictions per video sample. No sperm tracking or bounding boxes are required to solve this task. For evaluation, we apply the same principals as described under the prediction of motility task.

\subsection{Unsupervised Sperm Tracking Task}

Finding an optimal sperm for use in assisted reproduction can be crucial for fertilization success. However, detecting the "correct" spermatozoon can be difficult as they can move fast and be surrounded by spermatozoa and other debris. In the first optional task of the challenge, we ask participants to find the spermatozoon that moves faster than all others. This task requires that task participants track the spermatozoa. Within the tracked ones, the fastest is defined as either:

(1) Fastest average speed - The spermatozoon that moves the longest distance during the video (total distance/length of the video). This can then be calculated summarizing the different positions (in pixels) between each frame and divide on the number of frames.

(2) Highest top speed - The spermatozoon that has the highest average speed across the entire duration of the video. This can be calculated using the maximum of the differences between frames.

A challenge with this task is that the videos change view throughout the sample. This happens because the sample is moved below the microscope to observe the complete sample area. Therefore, the tracking has to be performed per viewpoint on the sample. We decided to keep the submission format to this task quite open, and leave it to the participants to deliver the information in the way that they see fit. Three separate andrology experts will do the evaluation, where they emphasize the correctness of the selected spermatozoa and how well the information is presented.

\subsection{Sperm Tracking Support Tool}

As previously mentioned, the current procedure for analyzing semen is through manual counting of spermatozoa, which is both tedious and time-consuming. To alleviate this process, we ask participants to come up with a tool that can assist medical experts better in observing and choosing sperms. This could be as simple as providing an interface with different filters that allow for better control of the tracking of spermatozoa. The tool's quality is decided via a majority vote from three medical experts in the field testing the tool. The assessment will focus on the usefulness and novelty of the idea (not user interface or usability in terms of human-computer interaction).

Submission to this task should be a GitHub repository containing the code and a short README containing setup and usage instructions for the submitted software. The submission will be evaluated by three separate andrology experts, who will assess how useful the software would be in a real-world clinic. Participating teams will get a report containing the final evaluation and a few pointers on where the software could be improved to be more clinically viable. 


\section{DISCUSSION AND OUTLOOK}

In this paper, we described the BioMedia 2020 challenge, which is the second part of a series of medical related challenges held at ACM Multimedia. This year, the challenge focuses on automatic semen analysis and the development of tools to aid clinicians in finding the optimal spermatozoa for fertilization. We presented four different tasks, two of which were optional, where participants were given an open dataset consisting of video recordings and related sensor data from 85 free-willing participants. Last year, five teams participated in BioMedia.

Only one team participated in the this years challenge. We believe multiple factors influenced the lack of participating teams this year. First of all, COVID-19 has and still is making a large impact on people's lives and their ability to work, which may have affected the number of people who were able to participate. Another possible reason for the lack of participation could be that assisted reproduction has certain cultural barriers that make it a difficult topic to work with $[2,5,13,19,21]$. This is also supported by the fact that a lot of people were interested in the task, but in the end, only one team submitted. Another limiting factor could be that this year the task was truly multimodal, and it requires a lot of effort to analyze data in a multimodal manner. BioMedia's primary purpose is to encourage multimedia researchers to explore the field of medical multimedia. In the future, we hope to continue the challenge over the next few years with different medical use-cases each year. The 2021 version will focus on mental health [12] using multimodal data from a variety of different data sources. In conclusion, we believe that the multimedia community can have a great impact on the field of medicine. We can already see several works in that direction to support our effort in encouraging even more researchers to follow that path.

\section{REFERENCES}

[1] Ying Bi, Bing Xue, and Mengjie Zhang. 2018. An Automatic Feature Extraction Approach to Image Classification Using Genetic Programming. In Applications of Evolutionary Computation, Kevin Sim and Paul Kaufmann (Eds.). 421-438.

[2] Henny M. W. Bos and Floor B. Van Rooij. 2007. The influence of social and cultural factors on infertility and new reproductive technologies. Journal of Psychosomatic Obstetrics \& Gynecology 28, 2 (2007), 65-68. https://doi.org/10.1080/01674820701447439

[3] Violeta Chang, Laurent Heutte, Caroline Petitjean, Steffen Härtel, and Nancy Hitschfeld. 2017. Automatic classification of human sperm head morphology. Computers in Biology and Medicine 84 (2017), 205 - 216. https://doi.org/10.1016/j.compbiomed.2017.03.029

[4] Trevor G. Cooper, Elizabeth Noonan, Kirsten M. Vogelsong, Michael T. Mbizvo, Sigrid von Eckardstein, Jacques Auger, H.W. Gordon Baker, Hermann M. Behre, Trine B. Haugen, Thinus Kruger, and Christina Wang. 2009. World Health Organization reference values for human semen characteristics ${ }^{*} \ddagger$. Human Reproduction Update 16, 3 (11 2009), 231-245. https://doi.org/10. 1093/humupd/dmp048 arXiv:http://oup.prod.sis.lan/humupd/articlepdf/16/3/231/1791304/dmp048.pdf

[5] Daisy Deomampo. 2019. Racialized Commodities: Race and Value in Human Egg Donation. Medical Anthropology 38, 7 (2019), 620-633. https://doi.org/10.1080/01459740.2019.1570188

[6] Karan Dewan, Tathagato Rai Dastidar, and Maroof Ahmad. 2018. Estimation of Sperm Concentration and Total Motility From Microscopic Videos of Human Semen Samples. In Proc. of CVPR Workshops.
[7] Muhammad Farooq and Edward Sazonov. 2017. Feature Extraction Using Deep Learning for Food Type Recognition. In Bioinformatics and Biomedical Engineering, Ignacio Rojas and Francisco Ortuño (Eds.). 464-472.

[8] Pål Halvorsen, Michael Alexander Riegler, and Klaus Schoeffmann. 2019. Medical Multimedia Systems and Applications. In Proc. of ACM MM. 2711-2713. https://doi.org/10.1145/3343031.3351319

[9] Trine B. Haugen, Steven A. Hicks, Jorunn M. Andersen, Oliwia Witczak, Hugo L. Hammer, Rune Borgli, Pål Halvorsen, and Michael Riegler. 2019. VISEM: A Multimodal Video Dataset of Human Spermatozoa. In Proc. of MMSYS. 261-266. https://doi.org/10.1145/3304109.3325814

[10] Steven Hicks, Michael Riegler, Pia Smedsrud, Trine B. Haugen, Kristin Ranheim Randel, Konstantin Pogorelov, Håkon Kvale Stensland, Duc-Tien Dang-Nguyen, Mathias Lux, Andreas Petlund, Thomas de Lange, Peter Thelin Schmidt, and Pål Halvorsen. 2019. ACM Multimedia BioMedia 2019 Grand Challenge Overview. In Proc. of ACM MM. 2563-2567. https://doi.org/10.1145/3343031.3356058

[11] Steven A Hicks, Jorunn M Andersen, Oliwia Witczak, Vajira Thambawita, Pål Halvorsen, Hugo L Hammer, Trine B Haugen, and Michael A Riegler. 2019. Machine Learning-Based Analysis of Sperm Videos and Participant Data for Male Fertility Prediction. Scientific Reports 9, 1 (2019), 16770. https://doi.org/10.1038/s41598-019-53217-y

[12] Petter Jakobsen, Enrique Garcia-Ceja, Lena Antonsen Stabell, Ketil Joachim Oedegaard, Jan Oystein Berle, Steven Hicks, Vajira Thambawita, Pål Halvorsen, Ole Bernt Fasmer, and Michael Alexander Riegler. 2020. Psykose: A Motor Activity Database of Patients with Schizophrenia. https://doi.org/10.31219/osf.io/e2tzf

[13] S. Kol. 2018. Ultra-Orthodox Jews and infertility diagnosis and treatment. Andrology 6, 5 (2018), 662-664. https://doi.org/10.1111/andr. 12533

[14] Mathias Lux, Michael Riegler, Pål Halvorsen, Konstantin Pogorelov, and Nektarios Anagnostopoulos. 2016. LIRE: Open Source Visual Information Retrieval. In Proc. of MMSYS. Article 30, 4 pages. https: //doi.org/10.1145/2910017.2910630

[15] F Pérez-sánchez, JJ de Monserrat, and C Soler. 1994. Morphometric analysis of human sperm morphology. International journal of andrology 17, 5 (1994), 248-255.

[16] Michael Riegler, Mathias Lux, Carsten Griwodz, Concetto Spampinato, Thomas de Lange, Sigrun L. Eskeland, Konstantin Pogorelov, Wallapak Tavanapong, Peter T. Schmidt, Cathal Gurrin, Dag Johansen, Håvard Johansen, and Pål Halvorsen. 2016. Multimedia and Medicine: Teammates for Better Disease Detection and Survival. In Proc. of ACM MM. 968-977. https://doi.org/10.1145/2964284.2976760

[17] Fariba Shaker, S Amirhassan Monadjemi, and Javad Alirezaie. 2017. Classification of human sperm heads using elliptic features and LDA. In 3rd International Conference on Pattern Recognition and Image Analysis (IPRIA). 151-155.

[18] H. Tamura, S. Mori, and T. Yamawaki. 1978. Textural Features Corresponding to Visual Perception. IEEE Transactions on Systems, Man, and Cybernetics 8, 6 (1978), 460-473. https://doi.org/10.1109/TSMC. 1978.4309999

[19] Andrea Whittaker, Marcia C. Inhorn, and Francoise Shenfield. 2019. Globalised quests for assisted conception: Reproductive travel for infertility and involuntary childlessness. Global Public Health 14, 12 (2019), 1669-1688. https://doi.org/10.1080/17441692.2019.1627479

[20] World Health Organization, Department of Reproductive Health and Research. 2010. WHO laboratory manual for the examination and processing of human semen.

[21] Sophie Zadeh. 2016. Disclosure of donor conception in the era of non-anonymity: safeguarding and promoting the interests of donorconceived individuals? Human Reproduction 31, 11 (10 2016), 24162420. https://doi.org/10.1093/humrep/dew240 